\title{
Photon-plasmon coupling for fundamental-mode phase-matched third harmonic and triplet photon generation
}

\author{
Tianye Huang, Member IEEE, Jianxing Pan, Zhuo Cheng, Chaolong Song, Jin Wang, Xuguang Shao, \\ Member IEEE, Perry Shum Ping, Senior Member IEEE, and Gilberto Brambilla
}

\begin{abstract}
On-chip third harmonic generation (THG) and its inverse process, namely the generation of entangled triplet photons with Greenberger-Horne-Zeilinger (GHZ) state, have attracted much interest in the last decade. To enhance the conversion efficiency or photon creation rate in optical waveguides, phase matching is a crucial condition which must be satisfied. However, material dispersion usually prevents the phase matching condition (PMC) between input pump and generated radiation when they are both guided in the fundamental modes. In this paper, a dielectric-loaded waveguide based on fundamental mode photon-plasmon coupling is proposed for efficient THG and triplet photon generation (TPG). Leveraging on the unique dispersion properties of transparent conductive oxide, the third harmonic radiation can be guided by the conventional photonic mode in the near-infrared, while the pump frequency is confined by surface plasmon polariton (SPP) mode in the mid-infrared. According to our simulations, the THG efficiency and TPG rate can achieve $\sim 10^{-4}$ and $32 \mathrm{~Hz}$, respectively. The proposed waveguide can be a promising platform for all-optical and quantum signal processing.
\end{abstract}

Index Terms-Harmonic generation, nonlinear signal processing, guided waves, quantum optics

\section{INTRODUCTION}

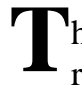
hird harmonic generation (THG) has attracted great research interests in recent years because of its wide range of application in various areas such signal processing [1], imaging [2], etc. Particularly, its inverse process, the triplet photon generation (TPG), provides a direct approach for spontaneous three-photon parametric down-conversion [3-10], in which a photon propagating in a nonlinear material

Manuscript received XXX, 2018.

This work was supported in part by the National Natural Science Foundation of China under Grant 61605179, in part by the Fundamental Research Funds for the Central Universities, China University of Geosciences (Wuhan) under grant 162301132703 and G1323511665. (Corresponding Author: Tianye Huang).

T. Huang, J. Pan, Z. Cheng, C. Song, and J. Wang are with School of Mechanical Engineering and Electronic Information, China University of Geosciences (Wuhan), Wuhan, China (Email: tianye_huang@163.com).

X. Shao and P. P. Shum is with Center of Fiber Technology, School of Electrical and Electronic Engineering, Nanyang Technological University, Singapore.

G. Brambilla is with Optoelectronics Research Centre, University of Southampton, Southampton, UK. spontaneously splits into three photons with energy conservation. Unlike the THG, TPG is quantum in nature [11] and represents a three-photon Greenberger-Horne-Zeilinger (GHZ) state [12], whose three-body quantum entanglement can be more generally employed for quantum optics experiments. Since both THG and TPG share the same phase matching condition (PMC) and nonlinear coupling coefficients, the efficiency of the two processes are strongly correlated.

Generally, both plasmonic and dielectric structures can be used to enhance the nonlinear effect [13-15]. Until now, various approaches had been proposed or experimentally demonstrated for THG/TPG, such as photonic crystal waveguide [16], plasmonic waveguide $[17,18]$, germanium-on-silicon waveguide [19], microring resonator [20], chalcogenide fiber [21], glass microfiber [22], and highly germania-doped fiber [23]. Although these are certainly significant steps on THG, direct implementation of TPG are still challenge for most of these approaches. One of the reasons is the utilization of inter-modal phase matching techniques which means the third harmonic radiation is propagating in a higher-order mode (HOM). Therefore, for the TPG process, the pump must be launched in this specific mode and thus an additional mode conversion is needed before the photon creation process. Generally speaking, it is rather difficult to excite the radiation mode in photonic crystal waveguides [16] or the slot mode in plasmonic waveguides [17, 18]. Furthermore, for HOMs, there always exist anti-phase field distribution, which contributes negatively to the overall mode overlap and consequently reduces the nonlinear coupling coefficients. To overcome this limitation, it is desired that the two fundamental modes at pump and harmonic can be phase-matched, namely intra-modal PMC. In [24], a hybrid photonic crystal fiber is designed for this purpose. In this fiber, leveraging on the photonic bandgap guiding mechanism, the phase-matched interaction can be occurred between the fundamental photonic mode at the pump frequency and the bandgap mode at the harmonic frequency. The fiber-based THG/TPG devices possess the advantage of long interaction length, but they are not suitable for on-chip planar integration.

Recently, transparent conductive oxide (TCO), such as indium cadmium oxide (CdO) and indium tin oxide (ITO) have gathered significant attentions as alternative materials for plasmonic and metamaterials applications. This is because the 
carrier concentration of these materials can be modified by heavily doping, electrical gating or post-deposition rapid thermal annealing processes so as to tailoring their complex refractive indices [25-28]. Furthermore, according to the dispersion curves, at longer wavelength such as in the mid-infrared (MIR), they behave like metals with the real part of their dielectric constants being negative, whereas at shorter wavelength such as in the near-infrared (NIR), they are more dielectric-like. However, currently the metallic feature seems to be more exciting since it plays significant roles in modulators and sensors. On the contrary, their dielectric property is not that attractive.

In this paper, for the first time, we exploit both NIR and MIR characteristics of TCO for THG/TPG. In the MIR, it supports surface plasmon polariton (SPP) modes, while in the NIR, a photonic mode is formed within the dielectric waveguide loaded on the $\mathrm{CdO}$ substrate. Due to the lifted wave vector of SPP mode in the MIR, the chromatic dispersion over triple frequency interval is overcome leading to fundamental-mode phase-matching between SPP mode and photonic mode for THG/TPG processes. This waveguide can be used for THG-based MIR-NIR up-conversion detection and TPG-based entangled photon generation in MIR within the atmospheric transparency window.

\section{CONCEPT AND WAVEGUIDE DESIGN}

The proposed structure is schematically shown in Fig. 1(a). It is a dielectric-loaded waveguide with $\mathrm{CdO}$ as substrate and the nonlinear organic material 2-[4dimethylamino)phenyl]-3-\{[4-( dimethylamino)phenyl] ethynyl \}buta-1,3-diene-1,1,4,4tetracar bonitrile (DDMEBT) as strip waveguide. For $\mathrm{CdO}$, the dielectric constant can be described according to the Drude model [28]

$$
\begin{aligned}
\varepsilon=\varepsilon^{\prime}+j \varepsilon^{\prime \prime} & =\varepsilon_{\infty}-\frac{\omega_{p}}{\omega(\omega+j \gamma)} \\
\omega_{p} & =\sqrt{\frac{n e^{2}}{m_{\mathrm{eff}} \varepsilon_{0}}}
\end{aligned}
$$

where $\varepsilon^{\prime}$ and $\varepsilon^{\prime \prime}$ are the real and imaginary parts of the permittivity, $\varepsilon_{\infty}$ is the high-frequency limit of the permittivity, $\omega_{p}$ is the plasma frequency, $\omega$ is the angular frequency of light and $\gamma$ is the damping rate. In Eq. (2), $n$ is the electron density, $e$ is the electron charge, $\varepsilon_{0}$ is the vacuum absolute permittivity and $m_{\text {eff }}$ is the electron effective mass. And the value of $n, e, \varepsilon_{0}$, $\gamma, m_{\text {eff }}$ and $\varepsilon_{\infty}$ are $2.8 \times 10^{26} \mathrm{~m}^{-3}, 1.602 \times 10^{-19} \mathrm{C}, 8.854 \times 10^{-12}$ $\mathrm{F} / \mathrm{m}, 2.92 \times 10^{13} \mathrm{rad} / \mathrm{s}, 1.823 \mathrm{e}^{-31} \mathrm{~kg}$ and $5.5 \mathrm{~F} / \mathrm{m}$, respectively.
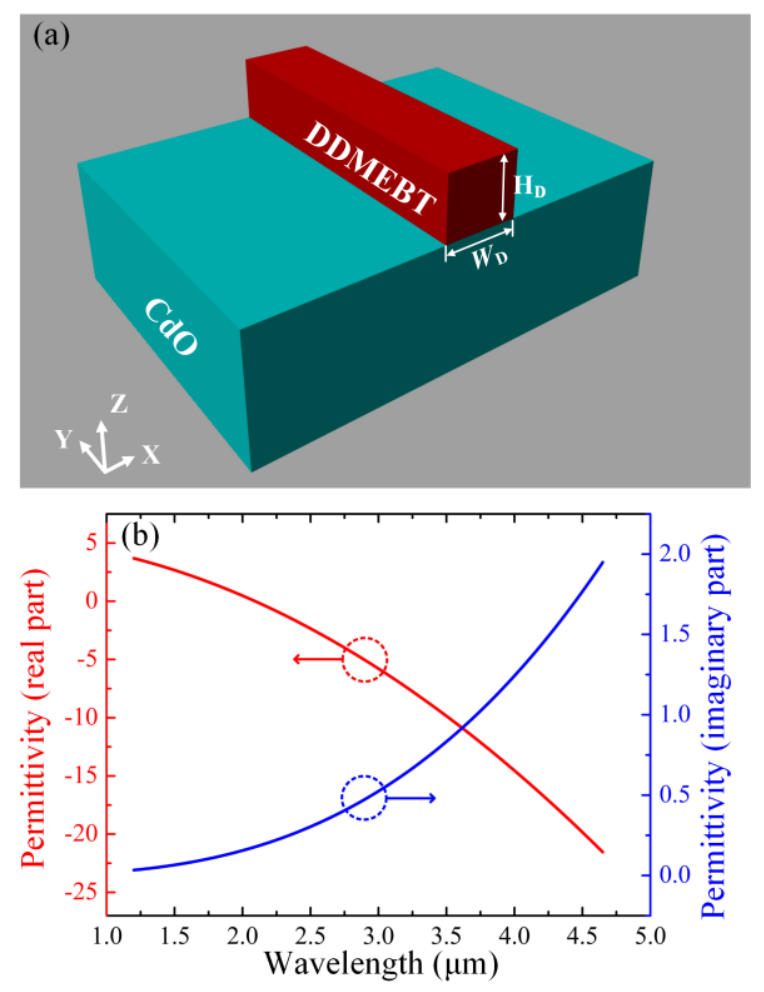

Fig. 1 (a) Schematic diagram of the THG/TPG waveguide, (b) CdO permittivity versus wavelength.

According to Eq. (1) and (2), the dispersion relation of $\mathrm{CdO}$ is highly dependent on the carrier density. A typical dispersion curve is demonstrated in Fig. 1(b). It is shown that the real part of the dielectric constant becomes negative from $\sim 2 \mu \mathrm{m}$, thus supporting plasmonic modes at longer wavelengths, while at short wavelengths, its value is positive with a small imaginary part thus behaving as dielectric material. Here we consider the interaction between modes at $1.55 \mu \mathrm{m}$ and $4.65 \mu \mathrm{m}$. The refractive indices of DDMEBT is 1.7973 and 1.7656 [29], respectively. According to Fig. 1(b), at $1.55 \mu \mathrm{m}$, the index of DDMEBT is larger than the substrate and a photonic mode can be formed within the waveguide due to total internal reflection. With waveguide height $\mathrm{H}_{\mathrm{D}}=1.4 \mu \mathrm{m}$, the effective indices of the plasmonic mode at $4.65 \mu \mathrm{m}$ and photonic mode at $1.55 \mu \mathrm{m}$ at different waveguide widths $\mathrm{W}_{\mathrm{D}}$ are demonstrated in Fig. 2(a). At $\mathrm{W}_{\mathrm{D}}=2.23 \mu \mathrm{m}$, the effective indices of the fundamental mode in the MIR and NIR are 1.7118+0.0176i and 1.7118+0.0015i, respectively, indicating that the PMC is satisfied. The corresponding electric mode profiles are illustrated in Fig. 2(b) and (c). Obviously, these two modes present typical features of surface plasmon and conventional total internal reflection, respectively, and therefore the third-order nonlinear coupling will happen between these two heterogeneous waves. Furthermore, the loss of the photonic mode is as low as $~ 0.05$ $\mathrm{dB} / \mu \mathrm{m}$, which is $\sim 14$ times lower than the one in inter-modal phase-matched plasmonic waveguide [17], in which both the pump and harmonic modes are in the form of plasmons.

The above-mentioned fundamental mode PMC is not an exception in this waveguide. By varying the structure parameters, different PMCs can be achieved, as shown in Fig. 3. With each waveguide height, there is a specific width for PMC. Particularly, it can be found that when the height is increased to 
$\sim 1.4 \mu \mathrm{m}$, the target width becomes less sensitive to height indicating larger fabrication-error tolerance.
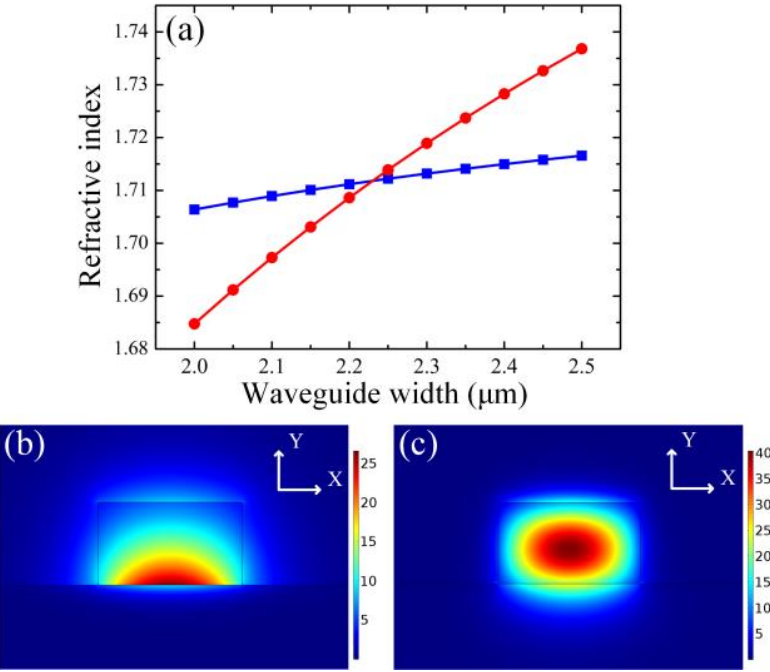

Fig. 2 (a) The effective indices of the plasmonic mode at $4.65 \mu \mathrm{m}$ and photonic mode at $1.55 \mu \mathrm{m}$ at different waveguide widths $\mathrm{W}_{\mathrm{D}}$ with $\mathrm{H}_{\mathrm{D}}=1.4 \mu \mathrm{m}$. Electric mode profiles of (b) plasmonic mode and (c) photonic mode at the phase matching condition.

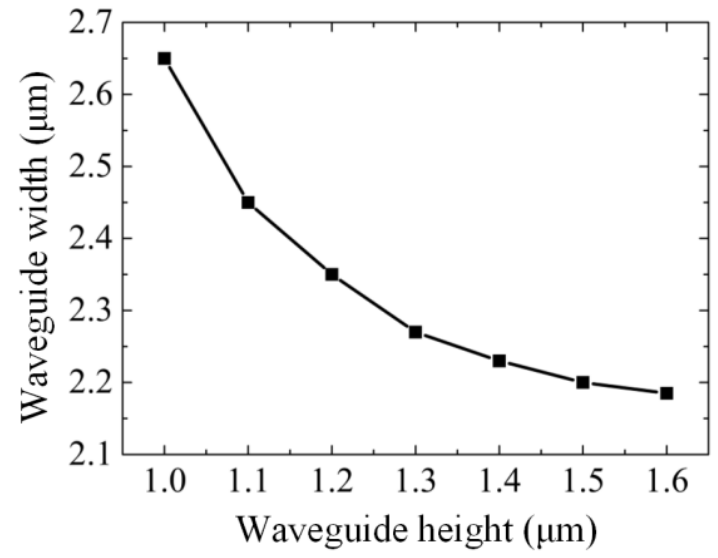

Fig. 3 The PMCs for different waveguide structures.

\section{THG PERFORMANCE IN THE PROPOSED WAVEGUIDE}

For the THG process, the performance is investigated by solving the coupled nonlinear Schrödinger equations [19],

$$
\begin{aligned}
& \frac{\partial E_{1}}{\partial z}+j \frac{1}{2} \beta_{2,1} \frac{\partial^{2} E_{1}}{\partial t^{2}}=-\frac{1}{2} \alpha_{1} E_{1}+ \\
& j\left(\gamma_{11}\left|E_{1}\right|^{2}+2 \gamma_{13}\left|E_{3}\right|^{2}\right) E_{1}+j \gamma_{1} E_{3} E_{1}^{*} E_{1}^{*} e^{j \Delta k z} \\
& \frac{\partial E_{3}}{\partial z}+\Delta \beta_{1} \frac{\partial E_{3}}{\partial t}+j \frac{1}{2} \beta_{2,3} \frac{\partial^{2} E_{3}}{\partial t^{2}}=-\frac{1}{2} \alpha_{3} E_{3}+ \\
& j\left(\gamma_{33}\left|E_{3}\right|^{2}+2 \gamma_{31}\left|E_{1}\right|^{2}\right) E_{3}+j \gamma_{3} E_{1} E_{1} E_{1} e^{-j \Delta k z}
\end{aligned}
$$

where $E_{1}, E_{3}$ are the complex amplitudes of pump and harmonic, $\Delta \beta_{1}=\beta_{1,3}-\beta_{1,1}$ denotes the group velocity mismatch, $\beta_{1,1}, \beta_{1,3}$ are the inverse of group velocity of pump and harmonic, respectively, $\Delta k=k_{3}-3 k_{1}$ is the phase mismatch, $\beta_{2,1}, \beta_{2,3}$ are the group velocity dispersion of pump and harmonic, respectively, $\gamma_{11}, \gamma_{13}, \gamma_{33}$, and $\gamma_{31}$ are the nonlinear phase modulation coefficients and $\gamma_{1}$ and $\gamma_{3}$ are the coefficients for THG. The nonlinear refractive indices of $\mathrm{CdO}$ and DDMEBT are $2.4 \times 10^{-15} \mathrm{~m}^{2} \cdot \mathrm{W}^{-1}$ and $8.5 \times 10^{-18} \mathrm{~m}^{2} \cdot \mathrm{W}^{-1}$, respectively [30, 31]. Under the condition of $\mathrm{H}_{D}=1.4 \mu \mathrm{m}$ and $\mathrm{W}_{D}=2.23 \mu \mathrm{m}$, the corresponding parameters are summarized in Table I.

TABLE I

\section{WAVEGUIDE PHYSICAL PARAMETERS}

\begin{tabular}{|c|c|c|c|}
\hline$\beta_{1,1}\left(\mathrm{~s} \cdot \mathrm{m}^{-1}\right)$ & $8.606 \times 10^{-9}$ & $\beta_{1,3}\left(\mathrm{~s} \cdot \mathrm{m}^{-1}\right)$ & $6.852 \times 10^{-9}$ \\
\hline$\beta_{2,1}\left(\mathrm{~s}^{2} \cdot \mathrm{m}^{-1}\right)$ & $1.103 \times 10^{-23}$ & $\beta_{2,3}\left(\mathrm{~s}^{2} \cdot \mathrm{m}^{-1}\right)$ & $1.056 \times 10^{-24}$ \\
\hline$\gamma_{11}\left(\mathrm{~m}^{-1} \cdot \mathrm{W}^{-1}\right)$ & 95.050 & $\gamma_{13}\left(\mathrm{~m}^{-1} \cdot \mathrm{W}^{-1}\right)$ & 15.924 \\
\hline$\gamma_{33}\left(\mathrm{~m}^{-1} \cdot \mathrm{W}^{-1}\right)$ & 58.382 & $\gamma_{31}\left(\mathrm{~m}^{-1} \cdot \mathrm{W}^{-1}\right)$ & 47.771 \\
\hline$\gamma_{1}\left(\mathrm{~m}^{-1} \cdot \mathrm{W}^{-1}\right)$ & 16.131 & $\gamma_{3}\left(\mathrm{~m}^{-1} \cdot \mathrm{W}^{-1}\right)$ & 16.131 \\
\hline
\end{tabular}

Firstly, the THG performance under pulsed pump is investigated with a peak power of $100 \mathrm{~W}$ and pulse width of 100 fs. Such pump wave can be obtained by using PPLN-based optical parametric oscillator [32]. Fig. 4(a) plots the conversion efficiency along the waveguide length. The conversion efficiency reaches maximum of $1.72 \times 10^{-4}$ after propagating $\sim 34 \mu \mathrm{m}$. After this well-determined propagation length, the pump power drops thereby so inducing a parametric gain not large enough to compensate the harmonic loss, and then a deamplification manifests itself resulting in lower efficiency. The generated harmonic peak power dependence on the pump peak power and their corresponding waveguide lengths are demonstrated in Fig. 4(b). The output power roughly satisfies the third-order exponential relation with input pump and the peak position also decreases with enhanced pump. The conversion at different PMC geometries are shown in Fig. 4(c), it can be found that the peak conversion efficiency can always be maintained above $1 \times 10^{-4}$, indicating a large flexibility in the fabrication. The optimal waveguide height is $\sim 1.4 \mu \mathrm{m}$. However, with waveguide higher than $1.3 \mu \mathrm{m}$, the achieved efficiency is quite close.

Next, we consider the condition when the waveguide is pumped by a continuous wave $(\mathrm{CW})$ with $2 \mathrm{~W}$ power as the same of germanium on silicon (Ge-on-Si) waveguide with cross section of $2.92 \mu \mathrm{m} \times 5.79 \mu \mathrm{m}$, bulk material loss coefficients of $1.5 \mathrm{~dB} / \mathrm{cm}$, effective area of $9.82 \mu \mathrm{m}^{2}$, and phase mismatch of $-5.15 \times 10^{5} \mathrm{~m}^{-1}$ [19]. According to Fig. 5, an output TH power of $28.8 \times 10^{-2} \mu \mathrm{W}$ is obtained with transmission length of $38.1 \mu \mathrm{m}$. In our case, though the linear phase mismatch $\Delta k$ is designed to be zero, the THG process still suffers from the nonlinear phase mismatch, which can be estimated by $\kappa_{\mathrm{THG}}=\Delta k+\left(2 \gamma_{31}-3 \gamma_{11}\right) P_{\mathrm{MIR}}$, where $P_{\mathrm{MIR}}$ is the THG pump power. A scale length $L_{\text {coh }}$, also named as the coherence length, can be defined as $L_{\mathrm{coh}}=\pi /\left|\kappa_{\mathrm{THG}}\right|$, so as to estimate the largest value of the waveguide length after which the THG process becomes suppressed. The $L_{\text {con }}$ with $2 \mathrm{~W}$ power is $\sim 0.82 \mathrm{~cm}$. Comparing to the transmission length with peak efficiency showing in Fig. 5, the $L_{\mathrm{coh}}$ is far less than the latter which means the intrinsic loss of the waveguide is dominated in the THG process. Nevertheless, such conversion and corresponding waveguide length are $\sim 24$ times and $\sim 1 / 350$ that of the Ge-on-Si waveguide when considering two fundamental 
modes interaction indicating much more power-efficient and compact footprint.
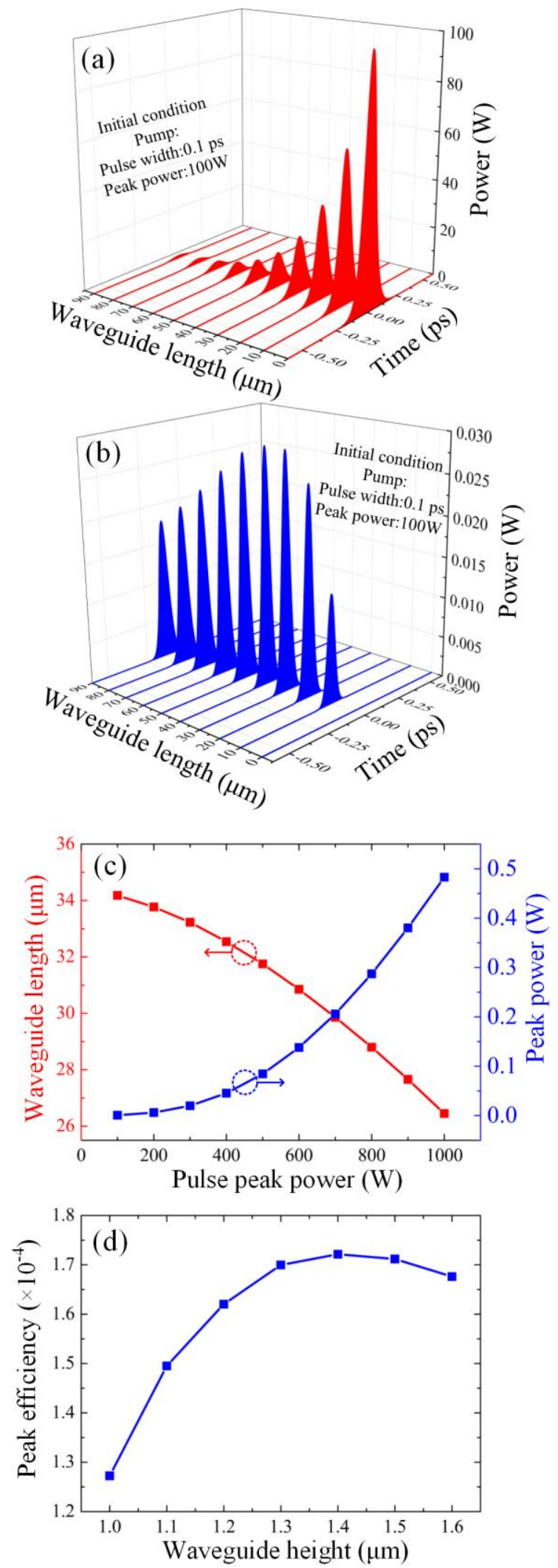

Fig. 4 Optical pulse evolutions along the waveguide with $0.1 \mathrm{ps}$, 100W pump pulse. (a) Pump pulse power (b) TH pulse power (c) peak power and waveguide length with respect to different peak power of pulse, and (d) the efficiency under different PMC geometries.

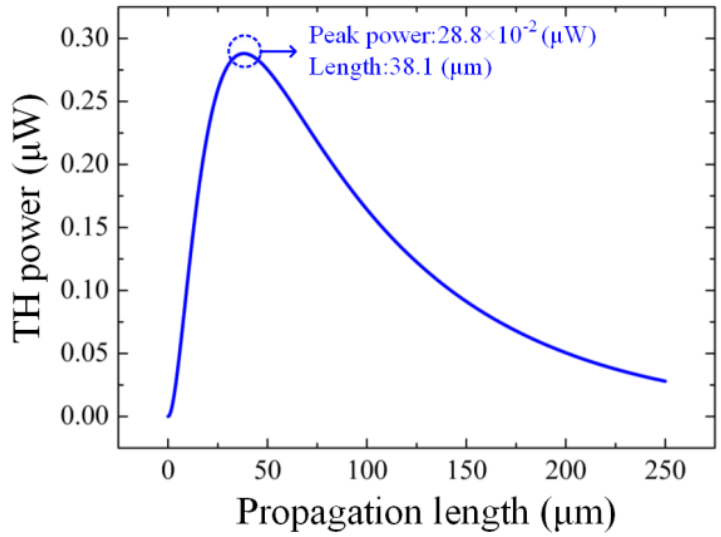

Fig. $5 \mathrm{TH}$ power with respect to the waveguide length pumped by a CW with 2 W power.

The fabrication-error tolerance and wavelength-shift tolerance is highly related to the phase mismatch $\Delta k$. Fig. 6 illustrates conversion contour plot with respect to the phase mismatch and propagation distance. Considering $3 \mathrm{~dB}$ conversion variation range, the mismatch tolerance is $-1.45 \times 10^{5} \mathrm{~m}^{-1}$ to $1.19 \times 10^{5} \mathrm{~m}^{-1}$, which roughly corresponds to variations of waveguide height, waveguide width and pump wavelength of $\pm 425 \mathrm{~nm}, \pm 355 \mathrm{~nm}$, and $\pm 249 \mathrm{~nm}$, respectively. Therefore, the proposed waveguide exhibits the advantages of strong fabrication-error tolerance and wavelength-shift tolerance, respectively.

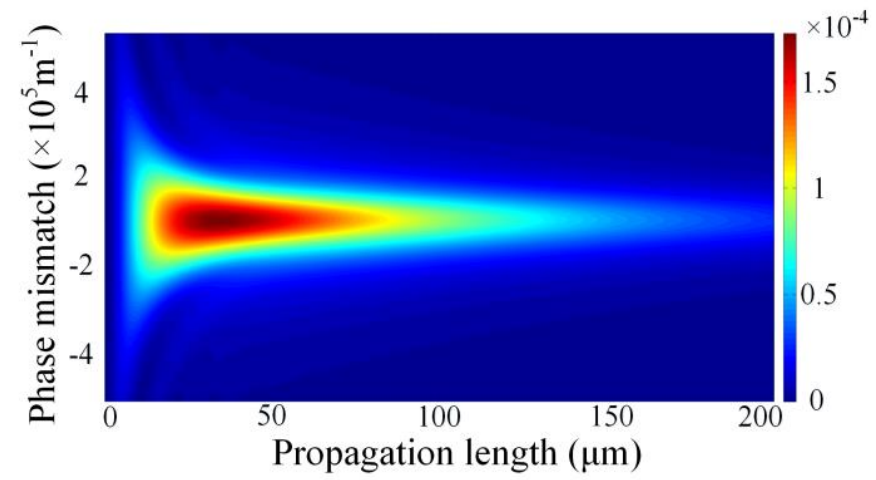

Fig. 6 The conversion efficiency with respect to the phase mismatch and propagation length.

\section{TPG PERFORMANCE IN THE PROPOSED WAVEGUIDE}

The results presents above show that the proposed waveguide is suitable for efficient THG. Note that in this configuration, the harmonic radiation is in the fundamental photonic mode. This feature makes it a promising candidate for the reversed process, namely TPG. Unlike other waveguides based on inter-modal phase matching, the pump beam can be easily coupled without the requirement of complex mode conversion. Additionally, by pumping at fiber communication wavelength of $1.55 \mu \mathrm{m}$, the wavelength of the generated triplet photon matches the atmospheric window transparency in the MIR, which can be a potential bridge between fiber-optic classic and free-space quantum communications. The TPG rate spectral density is given by [5] 


$$
d^{2} \Gamma \propto \frac{k_{r}^{\prime} \omega_{r}}{n_{r}^{2}} \frac{k_{s}^{\prime} \omega_{s}}{n_{s}^{2}} \frac{k_{i}^{\prime} \omega_{i}}{n_{i}^{2}} \sin c^{2}\left(\frac{\kappa z}{2}\right) d \omega_{r} d \omega_{s}
$$

where $\kappa=\Delta k+\left(\gamma_{33}-6 \gamma_{13}\right) P, P$ is the pump power, and $\omega_{r, s, i}, k_{r, s, i}^{\prime}$, and $n_{r, s, i}$, are the angular frequency, first-order derivative, and indices of the triplet photons, respectively, which must satisfy the energy conservation $\omega_{p}=\omega_{r}+\omega_{s}+\omega_{i}$. By using a six-order polynomial fitting for the propagation constants and their first-order derivative, the calculated normalized triplet photon spectral density is shown in Fig. 7(a). The $\mathrm{x}$-axis and y-axis indicate the wavelengths where any two generated photons locate during TPG process. It can be found that the spectral density map is slightly different from the conventional one with nearly elliptical shape $[3,24]$. This can be attributed to the strong dispersion of the plasmonic mode in the MIR. With a single frequency pump power of $200 \mathrm{~mW}$, Fig. 7(b) plots the triplet generation rate with different interaction length. Since the waveguide considered here is short, a nearly linear relation between TPG rate and length is obtained. However, with longer waveguides, the loss will limit the further increment of the TPG rate. With $30 \mu \mathrm{m}$ waveguides, we estimate a TPG rate of $\sim 32$ $\mathrm{Hz}$, which is even higher than the fiber-based TPG device with centimeter length [24].

\section{DisCUSSION AND CONCLUSION}

It should be mentioned that compared to NIR detectors, those in the spectral ranges of MIR are more expensive and inefficient $[33,34]$. Particularly, from the viewpoint of TPG, the single photon detector at MIR is still not available at this moment. However, with the fast development of quantum communication and probe technologies, extending the single photon detection to the MIR is an inevitable trend. We believe there will be a breakthrough in this area in the near future. It is also known that, in the MIR, there are a number of applications such as chemical or gas sensing [35, 36], biomedical application [37], and laser identification detection and ranging (LIDAR) [38]. Alternatively, a low-noise MIR detector can be implemented with a NIR detector and harmonic generation from MIR to NIR [39]. Therefore, to facilitate such applications, from the viewpoint of THG, the proposed waveguide provides a promising platform for upconversion detection. Comparing to the second harmonic generation (SHG), benefit from the triple frequency interval between pump and harmonic, upconversion detection can be realized in even longer wavelength. Note that, though only $1.55 \mu \mathrm{m}-4.65 \mu \mathrm{m}$ interaction is discussed in this paper, the proposed approach can easily extend to other MIR wavelength by fabricating $\mathrm{CdO}$ substrate with proper carrier density and designing the waveguide geometries, simultaneously.

For fabrication, the bottom $\mathrm{CdO}$ can be prepared by reactive high-power impulse magnetron sputtering (HiPIMS) and the detail fabrication process is descripted in [28]. The DDMEBT waveguide can be fabricated by organic molecular beam deposition in a high vacuum with proper pressure, temperature, and deposition rate [29] followed by E-beam lithography and plasma etching [40].

In summary, we have proposed a dielectric-loaded waveguide for THG/TPG. Based on the unique characteristics of transparent conductive oxide $\mathrm{CdO}$, the PMC between the fundamental plasmonic mode in the MIR and the photonic mode in the NIR is achieved. By optimizing the waveguide structure, the THG process in this waveguide is investigated in detail. More importantly, the fundamental photonic mode nature at the harmonic wavelength makes the waveguide a promising platform for integrated TPG. According to our simulations, a TPG rate up to $32 \mathrm{~Hz}$ can be achieved under 200 $\mathrm{mW}$ pump. The proposed structure can play a significant role in the areas of on-chip MIR up-conversion detection, wavelength conversion, and quantum signal processing.
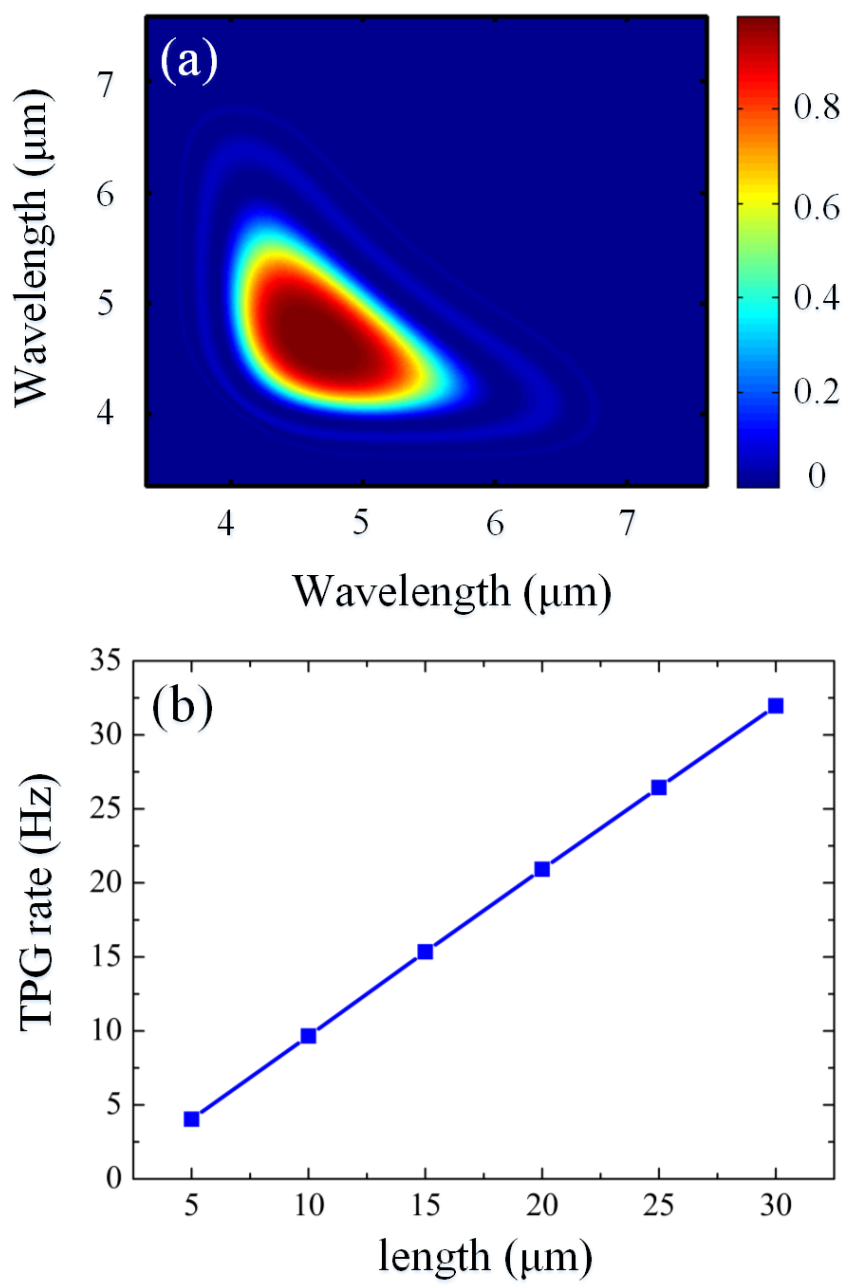

Fig. 7 (a) Normalized spectral density of TPG rate, and (b) TPG rate as a function of the waveguide length with the pump power of $200 \mathrm{~mW}$ at $1.55 \mu \mathrm{m}$.

\section{REFERENCES}

[1] C. Monat, C. Grillet, M. Collins, A. Clark, J. Schroeder, C. Xiong, J. Li, L. O'Faolain, T. F. Krauss, B. J. Eggleton, and D. J. Moss, "Integrated optical auto-correlator based on third-harmonic generation in a silicon photonic crystal waveguide," Nat. Commun., vol. 5, no. 3246, pp. 1-22, 2014.

[2] Y. C. Chen, S. Y. Lee, Y. Wu, K. Brink, D. B. Shieh, T. D. Huang, R. R. Reisz, and C. K. Sun, "Third-harmonic generation microscopy reveals dental anatomy in ancient fossils," Opt. Lett., vol. 40, no. 7, pp. 1354-1357, 2015.

[3] M. Corona, K. G. Palmett, and A. B. U'Ren, "Experimental proposal for the generation of entangled photon triplets by third-order spontaneous parametric downconversion in optical fibers," Opt. Lett., vol. 36, no. 2, pp. 190-192, 2011. 
[4] S. Richard, K. Bencheikh, B. Boulanger, and J. A. Levenson, "Semiclassical model of triple photons generation in optical fibers," Opt. Lett., vol. 36, no. 15, pp. 3000-3002, 2011.

[5] M. Corona, K. Garay-Palmett, and A. B. U'Ren, "Third-order spontaneous parametric down-conversion in thin optical fibers as a photon-triplet source," Phy. Rev. A, vol. 84, no. 3, pp. 033823, 2011.

[6] D. R. Hamel, L. K. Shalm, H. Hübel, A. J. Miller, F. Marsili, V. B. Verma, R. P. Mirin, S. W. Nam, K. J. Resch, and T. Jennewein, "Direct generation of three-photon polarization entanglement," Nat. Phot., vol. 8, pp. 801-807, 2014

[7] H. Hu bel, D. R. Hamel, A. Fedrizzi, S. Ramelow, K. J. Resch, and T. Jennewein, "Direct generation of photon triplets using cascaded photon-pair sources," Nature, vol. 466, no. 7306, pp. 601-603, 2010.

[8] A. Borne, T. Katsura, C. Félix, B. Doppagne, P. Segonds, K. Bencheikh, J. A. Levenson, and B. Boulanger, "Anisotropy analysis of third-harmonic generation in a germanium-doped silica optical fiber," Opt. Lett., vol. 40, no. 6, pp. 982-985, 2015

[9] A. Bétourné, Y. Quiquempois, G. Bouwmans, and M. Douay, "Design of a photonic crystal fiber for phase-matched frequency doubling or tripling," Opt. Express, vol. 16, no. 18, pp. 14255-14262, 2008.

[10] F. Shafiei, P. Srinivasan, and Z. Y. Ou, "Generation of three-photon entangled state by quantum interference between a coherent state and parametric down-conversion," Phys. Rev. A, vol. 70, pp. 043803, 2004.

[11] M. V. Chekhova, O. A. Ivanova, V. Berardi, and A. Garuccio, "Spectral properties of three-photon entangled states generated via three-photon parametric down-conversion in a $\chi^{(3)}$ medium," Phys. Rev. A, vol. 72, pp. $023818,2005$.

[12] A. Zeilinger, D. M. Greenberger, and M. A. Horne, "Bell's theorem without inequalities and beyond," Quantum Measurements in Optics, vol. 281, pp. 369-375, 1992.

[13] H. H. Hsiao, A. Abass, J. Fischer, R. Alaee, A. Wickberg, M. Wegener, and C. Rockstuhl, "Enhancement of second-harmonic generation in nonlinear nanolaminate metamaterials by nanophotonic resonances," Opt. Express, vol. 24, no. 9, pp. 9651-9659, 2016.

[14] K. Wang, L. Chen, H. Zhang, H. H. Hsiao, D. P. Tsai, and J. Chen, "Plasmon-enhanced optical nonlinearity for femtosecond all-optical switching," Appl. Phys. Lett., vol. 111, no. 18, pp. 181102, 2016.

[15] H. H. Hsiao, S. M. Chiou, Y. P. Chang, and H. C. Chang, "Broadly tuning resonant wavelengths of contour bowtie nano-antennas operating in the near- and mid-infrared," IEEE Photonics J., vol. 7, no. 4, pp. 4501108 , 2015

[16] B. Corcoran, C. Monat, C. Grillet, D. J. Moss, B. J. Eggleton, T. P. White, L. O'Faolain, and T. F. Krauss, "Green light emission in silicon through slow-light enhanced third-harmonic generation in photonic-crystal waveguides," Nat. Photon., vol. 3, no. 4, pp. 206-210, 2009.

[17] T. Wu, P. P. Shum, Y. Sun, X. Shao, and T. Huang, "Study on the crucial conditions for efficient third harmonic generation using a metal-hybrid-metal plasmonic slot waveguide," Opt. Express, vol. 23, no.1, pp. 253-263, 2015

[18] T. Huang, X. Shao, P. P. Shum, T. Lee, T. Wu, Z. Wu, Y. Sun, H. Q. Lam, J. Zhang, and G. Brambilla, "Internal Asymmetric Plasmonic Slot Waveguide for Third Harmonic Generation with Large Fabrication Tolerance," Plasmonics, vol. 11, no. 6, pp. 1451-1459, 2016.

[19] F. D. Leonardis, B. Troia, R. A. Soref, and V. M. N. Passaro, "Germanium-on-silicon waveguide engineering for third harmonic Generation in the Mid-Infrared," J. Lightw. Technol., vol. 33, no. 24, pp. 5103-5113, 2015

[20] S. Fujii, T. Kato, R. Suzuki, and T. Tanabe, "Third-harmonic blue light generation from Kerr clustered combs and dispersive waves," Opt. Lett., vol. 42, no. 10, pp. 2010-2013, 2017

[21] T. Cheng, R. Usaki, Z. Duan, W. Gao, D. Deng, M. Liao, Y. Kanou, M. Matsumoto, T. Misumi, T. Suzuki, and Y. Ohishi, "Soliton self-frequency shift and third-harmonic generation in a four-hole $\mathrm{As}_{2} \mathrm{~S}_{5}$ microstructured optical fiber," Opt. Express, vol. 22, no. 4, pp. 3740-3746, 2014.

[22] T. Lee, Y. Jung, C. A. Codemard, M. Ding, N. G. R. Broderick, and G. Brambilla, "Broadband third harmonic generation in tapered silica fibres," Opt. Express, vol. 20, no. 8, pp. 8503-8511, 2012.

[23] K. Bencheikh, S. Richard, G. Mélin, G. Krabshuis, F. Gooijer, and J. A. Levenson, "Phase-matched third-harmonic generation in highly germanium-doped fiber," Opt. Lett., vol. 37, no. 3, pp. 289-291, 2012.
[24] A. Cavanna, F. Just, X. Jiang, G. Leuchs, M. V. Chekhova, P. St.J. Russell, and N. Y. Joly, "Hybrid photonic-crystal fiber for single-mode phase matched generation of third harmonic and photon triplets," Optica, vol. 3, no. 9, pp. 952-955, 2016.

[25] K. Liu, C. R. Ye, S. Khan, and V. J. Sorger, "Review and perspective on ultrafast wavelength-size electro-optic modulators," Laser Photon. Rev., vol. 9, no. 2, pp. 172-194, 2015.

[26] H. W. Lee, G. Papadakis, S. P. Burgos, K. Chander, A. Kriesch, R. Pala, U. Peschel, and H. A. Atwater, "Nanoscale conducting oxide PlasMOStor," Nano. Lett., vol. 14, no. 11, pp. 6463-6468, 2014.

[27] A. Capretti, Y. Wang, N. Engheta, and L. D. Negro, "Enhanced third-harmonic generation in Si-compatible epsilon-near-zero indium tin oxide nanolayers," Opt. Lett., vol. 40, no. 7, pp. 1500-1503, 2015.

[28] Y. Yang, K. Kelley, E. Sachet, S. Campione, T. S. Luk, J. P. Maria, M. B. Sinclair, and I. Brener, "Femtosecond optical polarization switching using a cadmium oxide-based perfect absorber," Nat. Photon., vol. 11, no. 6, pp. 390-395, 2017.

[29] M. T. Beels, M. S. Fleischman, I. Biaggio, B. Breiten, M. Jordan, and F. Diederich, "Compact TCBD based molecules and supramolecular assemblies for third-order nonlinear optics," Opt. Mater. Express, vol. 2, no. 3, pp. 294-303, 2012.

[30] V. Ganesh, Mohd. Shkir, S. AlFaify, I. S. Yahia, H. Y. Zahran, A. F. Abd El-Rehim, "Study on structural, linear and nonlinear optical properties of spin coated $\mathrm{N}$ doped $\mathrm{CdO}$ thin films for optoelectronic applications," $J$. Mol. Struct., vol. 1150, pp. 523-530, 2017.

[31] T. Wu, Y. Sun, X. Shao, P. P. Shum, and T. Huang, "Efficient phase-matched third harmonic generation in an asymmetric plasmonic slot waveguide," Opt. Express, vol. 22, no. 15, pp. 18612-18624, 2014.

[32] N. Coluccelli, H. Fonnum, M. Haakestad, A. Gambetta, D. Gatti, M. Marangoni, P. Laporta, and Gianluca Galzerano1, "250-MHz synchronously pumped optical parametric oscillator at 2.25-2.6 $\mu \mathrm{m}$ and

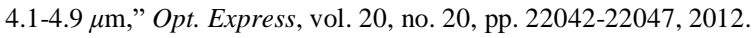

[33] S. D. Jackson, "Towards high-power mid-infrared emission from a fibre laser," Nat. Photon., vol. 6, no. 7, pp. 423-431, 2012.

[34] T.W. Neely, T. A. Johnson, and S. A. Diddams, "High-power broadband laser source tunable from $3.0 \mu \mathrm{m}$ to $4.4 \mu \mathrm{m}$ based on a femtosecond yb: fiber oscillator," Opt. Lett., vol. 36, no. 20, pp. 4020-4022, 2011.

[35] B. Mizaikoff, "Peer Reviewed: Mid-ir fiber-optic sensors," Anal. Chem., vol. 75, no. 11, pp. 258-A, 2003

[36] P. Werle, F. Slemr, K. Maurer, R. Kormann, R. Mücke, and B. Jänker, "Near-and mid-infrared laser-optical sensors for gas analysis," Opt. Laser. Eng., vol. 37, no. 2-3, pp. 101-114, 2002.

[37] M. J. Walsh, R. K. Reddy, and R. Bhargava, "Label-free biomedical imaging with mid-ir spectroscopy," IEEE J. Sel. Topics Quantum Electron., vol. 18, no. 4, pp. 1502-1513, 2012.

[38] S. E. Reutebuch, H.-E. Andersen, and R. J. McGaughey, "Light detection and ranging (lidar): an emerging tool for multiple resource inventory," $J$. Forest., vol. 103, no. 6, pp. 286-292, 2005.

[39] L. Høgstedt, J. S. Dam, A.-L. Sahlberg, Z. Li, M. Ald' en, C. Pedersen, and P. Tidemand-Lichtenberg, "Low-noise mid-ir upconversion detector for improved ir-degenerate four-wave mixing gas sensing," Opt. Lett., vol. 39, no. 18, pp. 5321-5324, 2014.

[40] T. Wu, P. P. Shum, X. Shao, Y. Sun, T. Huang, and L. Wei, "Efficient phase-matched third harmonic generation in a metal-clad plasmonic double-slot waveguide,” J. Opt., vol. 17, no. 2, pp. 025506, 2015. 